\title{
O que é mais eficiente para perda de peso: exercício contínuo ou intermi- tente? com ou sem dieta? uma revi- são baseada em evidências
}

\section{What is more effective for weight loss: continuous or intermittent exercise? with or without dieting? A review based on evidence}

\author{
Diego A. S. Silva ${ }^{1}$, Heloyse E. G. Nunes²
}

\begin{abstract}
RESUMO
Modelo do estudo: Revisão sistemática de literatura

Objetivo: Apresentar evidências científicas acerca do tipo de exercício aeróbio (Contínuo vs. Intermitente) que resulta em melhores efeitos na redução do peso corporal.

Metodologia: Foi realizada revisão da literatura nas bases de dados Pubmed, Medline, ISI Web of Knowledge, Ebsco Host e Scopus de artigos a partir do ano 2000.

Resultados: A combinação do exercício aeróbio e restrição calórica podem maximizar a perda de peso em indivíduos com sobrepeso. Ao comparar os dois tipos de exercícios aeróbios, a maior parte das evidências sugerem superioridade do intermitente em comparação ao contínuo na perda de peso.

Conclusão: Intervenções realizadas com dieta restrita combinadas com programas de exercícios realizados de três a seis vezes semanais, de preferência intermitente, em intensidade moderada a alta, apresentaram resultados mais significativos para perda de peso em comparação ao exercício contínuo.
\end{abstract}

Palavras-Chave: Exercício Físico; Exercício Aeróbico; Exercício Contínuo; Exercício Intermitente; Composição Corporal ; Peso Corporal.

\footnotetext{
ABSTRACT

Study design: Systematic literature review

Objective: To present scientific evidence about the type of aerobic exercise (Continuous vs. Intermittent) that results in better effects on reducing body weight.

Methodology: Literature review was performed on Pubmed, Medline, ISI Web of Knowledge, Ebsco Host and Scopus of articles from the year 2000.
}

1. Doutor em Educação Física, Professor adjunto da Universidade Federal de Santa Catarina, Núcleo de Pesquisa em Cineantropometria e Desempenho Humano, Florianópolis, Santa Catarina, Brasil.

2. Especialista em Treinamento Funcional, Universidade Federal de Santa Catarina, Núcleo de Pesquisa em Cineantropometria e Desempenho Humano, Florianópolis, Santa Catarina, Brasil.
Correspondência Diego Augusto Santos Silva Universidade Federal de Santa Catarina, Centro de Desportos, Departamento de Educação Física, Campus Universitário Reitor João David Ferreira Lima. CEP: 88040-900. Florianópolis, Santa Catarina, Brasil.

Artigo recebido em 06/06/2013 Aprovado para publicação em 15/09/2014 


\begin{abstract}
Results: The combination of aerobic exercise and caloric restriction can maximize weight loss in overweight individuals. By comparing the two types of aerobic exercises, most evidence suggests superiority of intermittent exercise compared to continuous exercise in weight loss.

Conclusion: Interventions performed with restricted diet combined with exercise programs performed from three to six times weekly, preferably intermittent, from moderate to high intensity, showed more significant results for weight loss compared to continuous exercise.
\end{abstract}

Keywords: Exercise, Physical; Exercise, Aerobic; Continuous Exercise; Intermittent Exercise; Body Composition; Body Weight.

\section{Introdução}

As projeções para os próximos 20 anos indicam que o número de indivíduos adultos com sobrepeso e obesidade em todo mundo aumentará em $44 \%$ e $45 \%$, respectivamente, totalizando 1,35 bilhões de pessoas com sobrepeso e 573 milhões de obesos em 2030. Se as recentes tendências seculares continuarem aumentando, os números absolutos podem subir ainda mais, para um total de 1,12 bilhões de indivíduos obesos, ou $20 \%$ da população adulta do mundo. ${ }^{1}$

A obesidade pode trazer sérias complicações à saúde, apresentando estreita relação com as doenças e agravos não transmissíveis à saúde. Esta relação acarreta preocupações para os órgãos governamentais de saúde, como a Organização Mundial de Saúde $^{2}$ e o Centro de Controle e Prevenção de Doenças dos Estados Unidos ${ }^{3}$ que investigam as melhores estratégias para a prevenção e tratamento da obesidade.

Dentre as estratégias de tratamento da obesidade destacam-se o exercício físico e a dieta que são recomendados por muitos especialistas ${ }^{2,3}$ como alterações necessárias para a redução do peso corporal e de fatores de riscos associados. Ademais, evidências têm demonstrado que o exercício físico realizado de maneira aeróbia é mais eficiente para a redução do peso corporal em pessoas com sobrepeso e obesidade. ${ }^{4,5}$

O exercício aeróbio pode desempenhar um papel significativo na perda de peso, devido ao seu poder de aumentar o gasto de calorias. ${ }^{6}$ Além disso, aumenta a eficiência do sistema cardiorrespiratório e, consequentemente, diminui as chances de comorbidades associadas à obesidade, como as doenças cardiovasculares. ${ }^{7}$ Relatou-se que o exercício aeróbio reduz os riscos cardiovasculares, principalmente, devido à sua capacidade de melhorar a função vascular em adultos saudáveis ${ }^{8}$, em pacientes com doenças cardiovasculares $^{9}$, e em pessoas obesas. ${ }^{10}$

Entretanto, controvérsias são encontradas na literatura para a prescrição do tipo de exercício aeróbio a indivíduos com excesso de peso. Alguns pesquisadores acreditam que o exercício realizado de maneira contínua (ritmo constante e exercício prolongado, realizado em uma única sessão), é mais eficiente para a perda de peso $^{11}$, outros apostam no exercício intermitente (sessões curtas de exercício, realizadas várias vezes ao longo do dia) ${ }^{12}$ e outros não reportam diferenças entre os dois tipos. ${ }^{13}$ Estas discordâncias no estado da arte acarretam divergências entre os profissionais que tratam pessoas com sobrepeso por meio do exercício físico.

Neste sentido, o presente estudo de revisão tem como objetivo trazer evidências científicas acerca desta temática e tentar elucidar estas dúvidas que ainda cercam a ciência da prescrição de exercício.

\section{Métodos}

Esse estudo caracteriza-se como uma revisão de literatura narrativa. Todas as referências foram retiradas das bases de dados Pubmed, Medline, ISI Web of Knowledge, Ebsco Host e Scopus, disponíveis na internet. Nessa perspectiva, foram buscados, primeiramente, artigos publicados a partir do ano 2000. Entretanto, estudos clássicos que alertaram para a temática foram desenvolvidos no final da década de 80 e inicio de 90 e incluídos na presente pesquisa. Como critério de inclusão, só foram incluídos artigos científicos, os quais deveriam ter a amostra de adultos $(\geq 20$ anos) e ser na língua inglesa. Para isso, palavras-chave como "Exercises", "Physical Exercise", "Aerobic Exercises", "Intermittent Exercise", "Continuous Exercise”, "Weight Loss", "Body Composition”, foram combinadas de diversas maneiras.

\section{Resultados}

As Tabelas descrevem as características (população, protocolo de treinamento e principais resultados) dos estudos revisados, sendo subdivididos con- 
forme o tipo de exercício (contínuo ou intermitente) e presença ou não de intervenção de dieta. A faixa etária compreendeu indivíduos adultos ( $\geq 20$ anos) de ambos os sexos, sendo em sua maioria sedentários e com excesso de peso. Os protocolos dos treinamentos empregados diferem em relação à intensidade, frequência semanal, duração de cada sessão e duração total da intervenção.

Na Tabela 1, encontram-se 10 estudos revisados (sendo oito intervenções e duas meta-análise) sobre exercício aeróbio contínuo sem intervenção de dieta. ${ }^{14-23}$ A Tabela 2 descreve as características de sete estudos revisados (sendo cinco intervenções e duas meta-análise) sobre exercício aeróbio contínuo com intervenção de dieta. ${ }^{24-30}$ A Tabela 3 apresenta três estudos revisados sobre exercício aeróbio intermitente sem intervenção de dieta. ${ }^{13,18,31}$ Apenas um estudo foi encontrado na base de dados sobre exercício aeróbio intermitente com intervenção de dieta (Tabela 4$)^{32}$ e dois estudos compararam o exercício aeróbio contínuo e intermitente (Tabela 5). ${ }^{12,33}$

\section{Discussão}

\section{Efeito do exercício aeróbio contínuo e aeróbio intermitente sem intervenção de dieta}

Pesquisadores reportaram o efeito do treinamento aeróbio contínuo e intermitente sem dieta na composição corporal, com redução do índice de massa corporal (IMC) e do percentual de gordura $(\% \mathrm{G})$ e aumento de massa magra. ${ }^{17,19,20,32}$ Ao analisar os estudos que realizaram intervenções de treinamento aeróbio contínuo, observou-se que os melhores resultados na perda de peso e de massa de gordura apresentou-se quando o período de treinamento era realizado acima de 12 semanas, com frequência semanal igual ou superior a três vezes, com intensidade de moderada a alta e duração igual ou superior a 30 minutos. ${ }^{15,17,21,23}$

A maioria dos estudos foi realizada em indivíduos sedentários, logo, as adaptações causadas pelo próprio treinamento aeróbio, como a melhora da capacidade em utilizar lipídios para produção de energia, podem ter contribuído para perda de peso. Quanto ao volume de treino, exercícios de maior duração e com intensidades mais altas produzem Excesso de Oxigênio Consumido Pós-exercício (EPOC) mais duradouro e de maior magnitude, resultando em maior gasto calórico após o exercício, ${ }^{34,35}$ e ainda, este tipo de exercício pode propiciar manutenção e aumen- to de massa isenta de gordura, que contribui para maior perda de peso e de gordura no treinamento de alta intensidade, como observado em alguns estudos. ${ }^{19,21}$ Em contrapartida, pesquisa realizada por Rosenkilde et. al., ${ }^{20}$ encontrou menor perda de peso e de massa de gordura em exercício com volume e intensidade alta, justificando o achado por meio das respostas compensatórias ocasionadas pelo treinamento de alta intensidade (como aumento do apetite e diminuição da taxa metabólica basal) que, consequentemente, podem interferir no balanço energético. Em relação à frequência do treinamento, um estudo comparou o treinamento realizado com diferentes frequências semanais na mudança de gordura corporal e concluiu que todos proporcionaram perda de massa de gordura, contudo a única diferença significativa entre os grupos foi para aqueles que se exercitaram quatro ou mais vezes por semana. ${ }^{15}$

Em estudos revisados nas bases de dados sobre intervenção com exercício aeróbio intermitente sem dieta não foi evidenciada perda de peso de modo satisfatório, observou-se que estes estudos realizaram exercícios com volume de 120 a 150 minutos por semana, que apesar de promover melhora da aptidão física relacionada à saúde e das condições metabólicas, parece ter sido insuficiente para promover perda de peso sem auxílio de dieta. ${ }^{18}$ Contudo, quando analisados, percebe-se que a perda de peso não era o foco principal destes estudos, visto que tendem a apresentar a hipótese de que exercícios intermitentes podem apresentar resultados similares aos exercícios contínuos para a saúde. ${ }^{13,18,31}$

Além da contribuição que o exercício aeróbio pode proporcionar na melhora da composição corporal de indivíduos com excesso de peso, a atividade física representa uma estratégia terapêutica para a melhora do controle glicêmico, da resistência a insulina e da aptidão física, pois muitos obesos apresentam limitações metabólicas e físicas. ${ }^{31}$ Assim, alguns pesquisadores relataram resultados positivos no perfil lipídico e melhora do VO2máx, ${ }^{13,22,23}$ e estes resultados podem ser justificados pelo fato da capacidade aeróbia estar associada diretamente com adequados níveis de pressão arterial e do perfil lipídico. Logo, indivíduos com excesso de peso que possuem baixa capacidade aeróbia tendem a apresentar maior risco cardiovascular e de doenças crônicas não degenerativas, então, o aprimoramento da capacidade aeróbia contribui para a redução desses fatores de risco que são propensos nesta população. ${ }^{36}$ 
Tabela 1: Efeito do exercício aeróbio contínuo sem intervenção de dieta na composição corporal de indivíduos adultos

\begin{tabular}{|c|c|c|c|c|}
\hline Estudo & População & $\begin{array}{l}\text { Frequência, duração } \\
\text { e intensidade }\end{array}$ & $\begin{array}{l}\text { Período de } \\
\text { treinamento }\end{array}$ & Resultados \\
\hline $\begin{array}{l}\text { Garrow } \\
\text { et al. }{ }^{14}\end{array}$ & $\begin{array}{l}\text { Meta-análise de } \\
\text { intervenções com } \\
\text { exercício físico para } \\
\text { modificação da } \\
\text { composição corporal. }\end{array}$ & $\begin{array}{l}\text { Variado, não-especifica- } \\
\text { do }\end{array}$ & $\begin{array}{l}\text { Variado, } \\
\text { não-especificado }\end{array}$ & $\begin{array}{l}\text { Sexo masculino: perda de } 3 \mathrm{~kg} \text { após } \\
\text { intervenção de } 30 \text { semanas. } \\
\text { Sexo feminino: perda de peso de } 1,4 \mathrm{~kg} \\
\text { após } 12 \text { semanas. }\end{array}$ \\
\hline
\end{tabular}

Willis $\quad 90$ indivíduos (ambos

et al. ${ }^{15}$ os sexos)

Knoepfli- 57 homens

lenzin

et al. ${ }^{16}$

$\begin{array}{ll}\begin{array}{l}\text { Pritchard } \\ \text { et al. } 17\end{array} & 21 \text { homens } \\ \text { Jacobsen } & 26 \text { mulheres } \\ \text { et al. }{ }^{18} & \\ \begin{array}{l}\text { Slentz } \\ \text { et al. }{ }^{19}\end{array} & \begin{array}{l}120 \text { indivíduos } \\ \text { (ambos sexos) }\end{array}\end{array}$

Rosenkilde 61 indivíduos (ambos et al. 20 sexos)

Hansen 50 homens

et al. ${ }^{21}$

Shaw

et al. 22

10 homens

Hansen

et al. ${ }^{23}$

Meta-análise de inter- 2-5x semana, 30 minutos, intensidade não especificada

$3 x$ semana, uma hora, $79 \%$ da FCmáx,
8 semanas

Redução na massa de gordura corporal, com diferença significativa entre os grupos que se exercitaram quatro ou mais vezes por semana.

Grupo futebol: perda de peso de 1,6 $\pm 1,8 \mathrm{~kg}$ e de gordura de 2,0 $\pm 1,5 \mathrm{~kg}$.

Grupo corrida: perda de peso de 1,6 $42,1 \mathrm{~kg}$ e perda de gordura de 1,6 $\pm 1,5 \mathrm{~kg}$. O futebol apresentou diferença estatística entre os grupos na perda de massa de gordura $(\mathrm{P}<0,01)$.

$3 x$ semana, 30 minutos, 12 meses $65-75 \%$ da FCmáx

(1)

$3 x$ semana, 30 minutos, 16 meses $60-75 \%$ da FCRes

3x semana, variadas intensidades e volumes.

3x semana Grupo 1: 30 minutos, intensidade moderada. Grupo 2: 60 minutos, intensidade alta.

3x semana

Grupo 1: 55 minutos, intensidade baixa a moderada (50\% VO2 pico).

Grupo 2: 40 minutos, intensidade moderada a alta (75\% vO2pico).

3x semana, 40 minutos, 6 meses $75 \%$ VO2 pico. venções com exercício físico em indivíduos com obesidade, síndrome metabólica, diabetes mellitus tipo 2 e doenças cardíacas.
Treinamento aeróbio contínuo, treinamento intervalado de alta intensidade e resistência muscular.
13 semanas

6 meses não-especificado resultados maiores nos aspectos clínicos.

Maior frequência de treinamento (acima de dois dias na semana) e de longa duração (acima de 38 semanas) é mais eficaz na redução do tecido adiposo e na melhoria do $\mathrm{VO}_{2}$ máx.

FCmáx: Frequência cardíaca máxima; FCres: Frequência cardíaca de reserva; $\mathrm{VO}_{2}$ máx: Consumo máximo de oxigênio; $\mathrm{VO}_{2}$ pico: Consumo de oxigênio de pico. 
Tabela 2: Efeito do exercício aeróbio contínuo com intervenção de dieta na composição corporal de indivíduos adultos.

\begin{tabular}{|c|c|c|c|c|}
\hline Estudo & População & $\begin{array}{l}\text { Frequência, duração } \\
\text { e intensidade }\end{array}$ & $\begin{array}{l}\text { Período de } \\
\text { treinamento }\end{array}$ & Resultados \\
\hline $\begin{array}{l}\text { Pavlou } \\
\text { et al. } 24\end{array}$ & 11 homens & $\begin{array}{l}\text { 3x semana, } 35 \text { a } 60 \\
\text { minutos, } 70-85 \% \text { da } \\
\text { FCmáx. }\end{array}$ & 8 semanas & $\begin{array}{l}\text { Grupo dieta e exercício: Perda de peso } \\
\text { média de } 12,0 \pm 1,0 \mathrm{~kg} \text {, Grupo dieta: } \\
\text { Perda de peso média de } 7,1 \pm 0,5 \mathrm{~kg} \text {. }\end{array}$ \\
\hline $\begin{array}{l}\text { Keim } \\
\text { et al. } 25\end{array}$ & 10 mulheres & $\begin{array}{l}6 x \text { semana, durações } \\
\text { variadas, intensidade } \\
\text { de } 65-85 \% \text { do } \mathrm{VO}_{2} \text { máx. }\end{array}$ & Não especificado & $\begin{array}{l}\text { Grupo dieta e exercício: Perda de peso } \\
\text { de } 13,1 \pm 0,7 \mathrm{~kg} \text {, Grupo exercício: } \\
\text { Perdeu em média de 5,6 } 50,6 \mathrm{~kg} \text {. }\end{array}$ \\
\hline $\begin{array}{l}\text { Miller } \\
\text { et al. } 26\end{array}$ & $\begin{array}{l}\text { Meta-análise de } 493 \\
\text { intervenções com } \\
\text { exercício físico e die- } \\
\text { ta para modificação da } \\
\text { composição corporal. }\end{array}$ & $\begin{array}{l}\text { Treinamento aeróbio } \\
\text { (caminhada ou } \\
\text { corrida). }\end{array}$ & $\begin{array}{l}\text { Variado, } \\
\text { não-especificado }\end{array}$ & $\begin{array}{l}\text { Grupo dieta: Perda de peso de } 10,7 \pm \\
0,5 \mathrm{~kg} \text {; Grupo exercício: Perda de peso } \\
\text { de } 2,9 \pm 0,4 \mathrm{~kg} \text {; Grupo dieta e exercício: } \\
\text { Perda de peso de } 11,0 \pm 0,6 \mathrm{~kg} \text {. }\end{array}$ \\
\hline $\begin{array}{l}\text { Volek } \\
\text { et al. }{ }^{27}\end{array}$ & $\begin{array}{l}22 \text { indivíduos (ambos } \\
\text { sexos). }\end{array}$ & $\begin{array}{l}\text { Ciclismo, corrida, cami- } \\
\text { nhada, remo foram pres- } \\
\text { crito conforme as reco- } \\
\text { mendações Colégio } \\
\text { Americano de Medicina } \\
\text { do Esporte. }\end{array}$ & 8 semanas & $\begin{array}{l}\text { Redução do IMC de } 30,8 \pm 7,0 \text { para } 29,2 \\
\pm 6,4 \mathrm{~kg} / \mathrm{m}^{2} \text {. Redução do percentual de } \\
\text { gordura em } 2,7 \% \text {. }\end{array}$ \\
\hline $\begin{array}{l}\text { Wallman } \\
\text { et al. }{ }^{28}\end{array}$ & $\begin{array}{l}24 \text { indivíduos (ambos } \\
\text { sexos). }\end{array}$ & $\begin{array}{l}3 \text { grupos (intervalado e } \\
\text { dieta, continuo e dieta, } \\
\text { só dieta) duração de } 30 \\
\text { minutos. }\end{array}$ & 8 semanas & $\begin{array}{l}\text { Os grupos INT e CON demonstraram } \\
\text { melhorias significativas para } \mathrm{VO}_{2} \text { máx. } \\
\text { Grupo INT teve maior efeito na perda de } \\
\text { gordura na região abdominal. }\end{array}$ \\
\hline Wu et al. ${ }^{30}$ & $\begin{array}{l}\text { Meta-análise de } 18 \\
\text { intervenções com } \\
\text { exercício físico e } \\
\text { dieta para modifica- } \\
\text { ção da composição } \\
\text { corporal. }\end{array}$ & $\begin{array}{l}\text { Variado, não-especifi- } \\
\text { cado }\end{array}$ & $\begin{array}{l}\text { Pelo menos } \\
6 \text { meses }\end{array}$ & $\begin{array}{l}\text { Grupo dieta e exercício teve perda de } \\
\text { peso de } 1,14 \mathrm{~kg} \text { (IC95\%: } 0,21-2,07) \\
\text { maior do que no grupo de dieta. }\end{array}$ \\
\hline
\end{tabular}

FCmáx: Frequência cardíaca máxima; $\mathrm{VO}_{2}$ máx: Consumo máximo de oxigênio; IMC: Índice de massa corporal. 
Tabela 3: Efeito do exercício aeróbio intermitente sem intervenção de dieta na composição corporal de indivíduos adultos.

\begin{tabular}{|c|c|c|c|c|}
\hline Estudo & População & $\begin{array}{c}\text { Frequência, duração e } \\
\text { intensidade }\end{array}$ & $\begin{array}{l}\text { Período de } \\
\text { treinamento }\end{array}$ & Resultados \\
\hline $\begin{array}{l}\text { Quinn } \\
\text { et al. }{ }^{13}\end{array}$ & $\begin{array}{l}20 \text { indivíduos } \\
\text { (ambos sexos). }\end{array}$ & $\begin{array}{l}\text { 4x semana, } 30 \text { minutos, } \\
70-80 \% \text { da FCRes. }\end{array}$ & 24 semanas & $\begin{array}{l}\text { Não provocou alterações significativas no peso } \\
\text { corporal e no percentual de gordura. } \\
\text { Grupo intermitente teve melhor aprimoramen- } \\
\text { to na aptidão cardiorrespiratória e no perfil do } \\
\text { que no grupo contínuo. }\end{array}$ \\
\hline $\begin{array}{l}\text { Jacobsen } \\
\text { et al. }{ }^{18}\end{array}$ & 26 mulheres & $\begin{array}{l}\text { 5x semana, } 30 \text { minutos } \\
\text { (caminhada), 50-65\% da } \\
\text { FCRes. }\end{array}$ & 16 meses & Não houve alterações na massa corporal. \\
\hline $\begin{array}{l}\text { Murphy } \\
\text { et al. }{ }^{31}\end{array}$ & 47 mulheres & $\begin{array}{l}5 x \text { semana, } 30 \text { minutos, } \\
70 \text { a } 80 \% \text { FCmáx. }\end{array}$ & 10 semanas & $\begin{array}{l}\text { Grupo intermitente teve uma perda de peso e } \\
\text { diminuição da circunferência da cintura signifi- } \\
\text { cativamente maior que grupo contínuo. A per- } \\
\text { da de massa de gordura foi semelhante entre } \\
\text { os grupos. }\end{array}$ \\
\hline
\end{tabular}

FCres: Frequência cardíaca de reserva; FCmáx: Frequência cardíaca máxima.

Tabela 4: Efeito do exercício aeróbio intermitente com intervenção de dieta na composição corporal de indivíduos adultos.

\begin{tabular}{|c|c|c|c|c|}
\hline Estudo & População & $\begin{array}{l}\text { Frequência, duração e } \\
\text { intensidade }\end{array}$ & $\begin{array}{l}\text { Período de } \\
\text { treinamento }\end{array}$ & Resultados \\
\hline $\begin{array}{l}\text { Schimidt } \\
\text { et al. }{ }^{32}\end{array}$ & 38 mulheres & $\begin{array}{l}3-5 \times \text { semana, } 30 \text { minutos, } \\
75 \% \text { da FCRes. } \\
4 \text { grupos: controle (só die- } \\
\text { ta), contínuo (CON) ( } 30 \text { mi- } \\
\text { nutos direto), intermitente } 1 \\
\text { (INT1) (duas sessões por } \\
\text { dia) e intermitente } 2 \text { (INT2) } \\
\text { (três sessões por dia). }\end{array}$ & 12 semanas & $\begin{array}{l}\text { Grupo controle: Aumento de peso corporal de } \\
0,06 \pm 0,2 \mathrm{~kg} \text {. } \\
\text { Grupo INT1: Perda de peso significativa } \\
\text { de } 3,0 \pm 1,3 \mathrm{~kg} \\
\text { Grupo INT2: Perda de peso significativa } \\
\text { de } 4,4 \pm 2,3 \mathrm{~kg} \\
\text { Grupo CONT: Perda de peso significativa } \\
\text { de } 2,7 \pm 1,1 \mathrm{~kg} .\end{array}$ \\
\hline
\end{tabular}

FCres: Frequência cardíaca de reserva.

Tabela 5: Comparação do efeito do exercício aeróbio intermitente vs aeróbio contínuo na composição corporal de indivíduos adultos.

\begin{tabular}{|c|c|c|c|c|}
\hline Estudo & População & $\begin{array}{l}\text { Frequência, duração e } \\
\text { intensidade }\end{array}$ & $\begin{array}{l}\text { Período de } \\
\text { treinamento }\end{array}$ & Resultados \\
\hline $\begin{array}{l}\text { Jakicic } \\
\text { et al. }{ }^{33}\end{array}$ & 56 mulheres & $\begin{array}{l}3-4 \times \text { semana, } 20-40 \text { minu- } \\
\text { tos, } 70 \% \text { da FCRes. }\end{array}$ & 20 semanas & $\begin{array}{l}\text { Grupo intermitente: Perda de peso média de } \\
8,9 \pm 5,3 \mathrm{~kg} \\
\text { Grupo contínuo: Perda de peso média de } \\
6,4 \pm 4,5 \mathrm{~kg} \text {. }\end{array}$ \\
\hline $\begin{array}{l}\text { Jakicic } \\
\text { et al. }{ }^{12}\end{array}$ & 73 mulheres & $20-40$ minutos & 18 meses & $\begin{array}{l}\text { Grupo contínuo: Perda de peso de } 5,8 \pm \\
7,1 \mathrm{~kg} \text {. } \\
\text { Grupo intermitente: Perda de peso de } 3,7 \\
\pm 6,6 \mathrm{~kg} \text {. }\end{array}$ \\
\hline
\end{tabular}

FCres: Frequência cardíaca de reserva. 
Em meta-análise realizada com indivíduos com obesidade, síndrome metabólica, diabetes mellitus tipo 2 e doenças cardíacas, demonstrou-se que alternar modalidades de treinamento (exercício aeróbio contínuo, intervalo e de resistência muscular) produz resultados maiores do que somente uma modalidade de exercício nos aspectos clínicos, e ainda, que maior frequência de treinamento (acima de dois dias na semana) e de longa duração (acima de 38 semanas) é mais eficaz na redução do tecido adiposo, em pacientes obesos, e na melhoria do $\mathrm{VO}_{2}$ máx, em pacientes com doença cardíaca. ${ }^{23}$ Ademais, a alta intensidade do treinamento intervalado tem um impacto maior sobre $\mathrm{VO}_{2}$ máx em pacientes com doença cardíaca, e sobre sensibilidade à insulina em pacientes com síndrome metabólica, mas não em massa de tecido adiposo em indivíduos obesos. ${ }^{23}$

A perda de peso em indivíduos com excesso de peso torna-se relevante no sentido de melhorar os indicadores de saúde, ${ }^{22}$ desta forma preconiza-se modificações de hábitos de vida. Assim, o exercício aeróbio intermitente, apesar de não ter produzido muito efeito na perda de peso, tende a ser indicado, pois com base em evidências, sessões curtas de exercício durante o dia parece ser facilmente incorporado no estilo de vida. ${ }^{33}$ Estudo realizado por Willis et. al. ${ }^{15}$ confirmou que a aderência ao exercício intermitente é maior do que o exercício contínuo nas primeiras 24 semanas do treinamento, visto que esse período parece ser determinante para a aderência ao exercício e modificação do estilo de vida. Ademais, exercícios de intensidade moderada são importantes nesta fase inicial, ressaltando, assim, a utilização do exercício intermitente como estratégia motivacional para indivíduos sedentários e com excesso de peso ${ }^{31}$

\section{Efeito do exercício aeróbio contínuo e intermitente com intervenção de dieta}

Muitos pesquisadores já sugeriram que quando se deseja perder peso, a combinação de dieta e exercício físico representa uma intervenção muito superior à dieta ou ao exercício aeróbio feito separadamente. ${ }^{24,37-39}$ Em geral, a combinação de dieta e exercícios aeróbios tanto contínuo ${ }^{12,24-26,30}$ como intermitente, ${ }^{12,32,33}$ resultou em perdas de peso corporal significativas quando comparado à intervenção isolada de exercício ou dieta.

Essa constatação é apoiada em uma meta-análise que extraiu dados de 493 estudos sobre mudan- ças na composição corporal por meio de diferentes tipos de intervenção. Os estudos utilizaram intervenções terapêuticas como dieta, exercício aeróbio, ou dieta mais exercício aeróbio. Os resultados demonstraram que a perda de peso individual chegou a 10,7 $\pm 0,5 \mathrm{~kg}$ para intervenções de dieta, $2,9 \pm 0,4 \mathrm{~kg}$ para intervenções de exercício e $11,0 \pm 0,6 \mathrm{~kg}$ para as intervenções dieta mais exercício. ${ }^{26}$ Outra meta-análise extraiu dados de 18 artigos (de 1966 a 2007) e os pesquisadores demonstraram que no grupo de intervenção de dieta e exercício houve uma perda de peso de 1,14 kg (IC95\%: 0,21-2,07) maior do que no grupo de intervenção de dieta. ${ }^{30}$

Os resultados desses estudos dão suporte à teoria de que a melhor maneira de maximizar a perda de peso em indivíduos com excesso de peso é utilizar uma intervenção que combine restrição calórica e exercício aeróbio. $\mathrm{Na}$ obesidade visceral, a dieta tem importante papel para redução de fatores de risco como doenças cardiovasculares e resistência a insulina, visto que eleva a perda de gordura visceral. ${ }^{21} \mathrm{Ou}-$ tros benefícios proporcionados pelo exercício associado à dieta é o aumento da massa muscular e perda de peso decorrente da perda de massa de gordura, enquanto a dieta isolada provoca perda de massa muscular, o exercício físico favorece o equilíbrio saudável entre massa de gordura e massa muscular. Em relação aos aspectos clínicos, apesar da dieta isolada reduzir colesterol total (CT) e lipoproteína de baixa densidade (LDL), o aumento de lipoproteína de alta densidade (HDL) só ocorreu a partir da combinação com o exercício. Além disso, a combinação de dieta e exercício proporcionou melhora expressiva na sensibilidade à insulina e elevação da Taxa Metabólica de Repouso (TMR) após exercício. ${ }^{30}$

Autores relataram que muitas vezes a perda de peso após dois anos de intervenção pode parecer pequena, mas isto ocorre devido a adaptação do organismo ao exercício e dieta, que pode contribuir para desmotivação na permanência de programas de longo prazo. ${ }^{40,41}$ Contudo, sabe-se que a perda de peso ponderal pode atuar na reversão de doenças associadas à obesidade, sendo que reduções de 5\% a 10\% do peso já é suficiente para modificar fatores de risco para saúde. Outro aspecto encontrado na literatura é que depois de finalizados os programas de intervenções muitos indivíduos recuperam o peso. Alerta-se, assim, a relevância de ações de monitoramento e incentivo para que estes indivíduos possam manter as modificações dos hábitos de vida. ${ }^{42,43,44}$ 


\section{Efeito do exercício aeróbio contínuo VS aeróbio intermitente}

Alguns estudos apresentaram a superioridade do exercício intermitente ao exercício contínuo na perda de peso, ${ }^{31,33,43}$ na diminuição da circunferência da cintura e perda de massa de gordura, ${ }^{31,43}$ na melhora da aptidão cardiorrespiratória e no perfil metabólico. ${ }^{13}$ Outros apoiaram a hipótese de que o exercício intermitente e o contínuo têm efeitos similares na aptidão física e perda de peso. ${ }^{12,32}$

Um grupo de 56 mulheres sedentárias e com sobrepeso foi submetido a 20-40 minutos de exercício aeróbio contínuo ou intermitente, de três a quatro vezes por semana, a $70 \%$ da Frequência Cardíaca de Reserva (FCRes) individual, por um período de 20 semanas. A ingestão calórica ficou restrita a 1200 $1500 \mathrm{kcal}$ por dia. Os pesquisadores demonstraram que apesar de terem sido identificadas melhoras similares na condição cardiorrespiratória dos dois grupos, as integrantes do grupo de exercícios intermitentes perderam, em média, $2,5 \mathrm{~kg}$ a mais do que as integrantes do grupo de exercícios contínuos, com uma média de perda de peso de $8,9 \pm 5,3 \mathrm{~kg}$ e $6,4 \pm 4,5 \mathrm{~kg}$, respectivamente. ${ }^{33}$ Em contrapartida, outra pesquisa realizou uma intervenção de longa duração (18 meses) com programa de dieta e exercícios aeróbios regulares, praticados por 20-40 minutos de maneira contínua ou intermitente. Do baseline até os 18 meses, houve perda de peso de 5,8 $\pm 7,1 \mathrm{~kg}$ e $3,7 \pm 6,6$ $\mathrm{kg}$ nos grupos de exercício contínuo e intermitente, respectivamente. De acordo com os pesquisadores, a recuperação de uma parte do peso que tinha sido perdido em seis meses, tenha ocorrido devido a um declínio da participação nos exercícios, assim como ao aumento da ingestão calórica de várias participantes. Esse resultado sugere que alguns indivíduos têm dificuldade em aderir a um programa de dieta e exercícios por um longo período de tempo sendo necessário a elaboração de estratégias para mantê-los no programa. ${ }^{12} \mathrm{E}$ ainda, pesquisadores que comparam o efeito do exercício aeróbio contínuo com aeróbio intermitente apoiam a hipótese de que ambos exercícios (contínuo e intermitente) têm o mesmo efeito na aptidão aeróbia e perda de peso, realizados com restrição calórica. $^{32}$

Apesar dos estudos serem geralmente inconclusivos quanto a uma perda maior ou menor de peso entre exercícios intermitentes ou contínuos, o conceito de EPOC sugere que os exercícios intermiten- tes resultem em maiores perdas de peso. O EPOC é definido como a elevação do consumo de oxigênio para além dos níveis de repouso após a finalização do exercício, ${ }^{45}$ e consiste em uma quebra da homeostase iniciada pelo exercício. Um período total de EPOC mais longo pressupõe que o metabolismo ficará acima dos níveis de repouso por mais tempo, o que, por fim, resultará em um aumento no gasto calórico. A afirmação de que o período total de recuperação pósprática de exercícios intermitentes é mais longo é defendida por Kaminsky et al., ${ }^{46}$ que observaram um EPOC combinado $117 \%$ mais alto em seis mulheres de peso normal, após duas sessões de 25 minutos de exercício, em comparação a uma única sessão contínua de 50 minutos de exercício, sendo que as três sessões foram realizadas a 70\% do VO2máx. Outros pesquisadores concluíram que os exercícios intermitentes elevaram significantemente o gasto de energia pósexercício e, portanto, quando foram praticados regularmente, o EPOC teve um efeito cumulativo capaz de transformar o peso corporal dos indivíduos. ${ }^{47}$

Além de que um EPOC mais longo resulta em um maior gasto calórico, os exercícios intermitentes podem também ser mais apropriados para populações inativas com excesso de peso, já que permitem que os indivíduos pratiquem exercícios de curta duração, em horários convenientes ao longo do dia. Esse fator pode aumentar a probabilidade dos indivíduos atingirem os níveis recomendados de exercício, ${ }^{48}$ e ao mesmo tempo, facilitar a inclusão do exercício aeróbio no estilo de vida. Outra consideração a ser feita é a de que os indivíduos obesos reclamam frequentemente do desconforto provocado por suor, que pode ocorrer com mais frequência como resultado de exercícios contínuos. ${ }^{49}$ Os exercícios intermitentes podem reduzir a taxa de suor e, consequentemente, resultar em uma maior aderência da população com excesso de peso ao programa de exercícios. Por fim, como o exercício é associado a melhoras no humor e autoestima, sugere que o exercício intermitente pode resultar em mais momentos positivos. ${ }^{50}$

\section{Conclusão}

Exercícios contínuos e intermitentes sem dieta apresentaram perda de peso modesta em relação à combinação com restrição calórica. Intervenções realizadas com dieta restrita de $1000-1500 \mathrm{kcal}$ por dia ou a redução de $50 \%$ do consumo normal, combinadas com programas de exercícios intermitentes reali- 
zados de três a seis vezes semanais em intensidade moderada a alta apresentaram resultados mais significativos para perda de peso em comparação ao exercício contínuo. Ademais, exercícios aeróbios intermitentes oferecem maior flexibilidade na realização de um programa diário de exercícios, além de gerar benefícios similares, senão melhores do que exercícios aeróbios contínuos com a mesma duração de tempo. Em indivíduos com excesso de peso e sedentário, o exercício intermitente pode ser utilizado no início do treinamento, pois parece apresentar maior aderência deste indivíduo à prática de exercícios, podendo ser visto como estratégia para modificar o estilo de vida sedentário e reduzir fatores de risco associados ao excesso de peso.

\section{Referências}

1. Kelly T, Yang W, Chen CS, Reynolds K, He J. Global burden of obesity in 2005 and projections to 2030. Int J Obes. 2008; 32: 1431-7.

2. World Health Organization. Global strategy on diet, physical activity and health. Food Nutr Bull. 2004; 25: 292-302.

3. Center for Disease Control and Prevention. Obesity: Halting the Epidemic by Making Health Easier. National Center for Chronic Disease Prevention and Health Promotion. Improving health and quality of life for all people. Atlanta; 2009.

4. Ryan AS, Nicklas BJ, Berman DM. Aerobic exercise is necessary to improve glucose utilization with moderate weight loss in women. Obesity (Silver Spring). 2006; 14: 1064-72.

5. Silverman NE, Nicklas BJ, Ryan AS. Addition of aerobic exercise to a weight loss program increases BMD, with an associated reduction in inflammation in overweight postmenopausal women. Calcif Tissue Int. 2009; 84: 257-65.

6. Seagle HM, Strain GW, Makris A, Reeves RS. Position of the American Dietetic Association: weight management. J Am Diet Assoc 2009; 109: 330-46.

7. Wei $\mathrm{M}$ et al. Relationship between low cardiorespiratory fitness and mortality in normal-weight, overweight, and obese men. JAMA. 1999; 282: 1547-53.

8. Green DJ, O'driscoll JG, Blanksby BA, Taylor RR. Effect of casting on forearm resistance vessels in young men. Med Sci Sports Exerc. 1997; 29: 1325-31.

9. Pierce GL, Schofield RS, Casey DP, Hamlin SA, Hill JA, Braith RW. Effects of exercise training on forearm and calf vasodilation and proinflammatory markers in recent heart transplant recipients: a pilot study. Eur J Cardiovasc Prev Rehabil. 2008; 15: 10-18.

10. Nassis GP, Papantakou K, Skenderi K, Triandafillopoulou M, Kavouras SA, Yannakoulia M, et al. Aerobic exercise training improves insulin sensitivity without changes in body weight, body fat, adiponectin, and inflammatory markers in overweight and obese girls. Metab. 2005; 54: 1472-9.

11. Miller ER, Erlinger TP, Young DR, Jehn M, Charleston J, Rhodes D, et al. Results of the Diet, Exercise, and Weight Loss Intervention Trial (DEW-IT). Hypertension. 2002; 40: 612-18.
12. Jakicic JM, Winters C, Lang W, Wing RR. Effects of intermittent exercise and use of home exercise equipment on adherence, weight loss, and fitness in overweight women: a randomized trial. JAMA. 1999; 282: 1554-60.

13. Quinn TJ, Klooster JR, Kenefick RW. Two short, daily activity bouts vs. one long bout: are health and fitness improvements similar over twelve and twenty-four weeks? J Strength Cond Res. 2006; 20: 130-5.

14. Garrow JS, Summerbell CD. Meta-analysis: effect of exercise, with or without dieting, on the body composition of overweight subjects. Eur J Clin Nutr. 1995; 49: 1-10.

15. Willis FB, Smith FM, Willis AP. Frequency of exercise for body fat loss: a controlled, cohort study. J Strength Cond Res. 2009; 23: $2377-80$.

16. Knoepfli-lenzin $\mathrm{C}$ et al. Effects of a 12-week intervention period with football and running for habitually active men with mild hypertension. Scand J Med Sci Sports. 2010; 20: 72-9.

17. Pritchard JE, Nowson CA, Wark JD. A worksite program for overweight middle-aged men achieves lesser weight loss with exercise than with dietary change. J Am Diet Assoc 1997; 97: 37-42.

18. Jacobsen DJ, Donnelly JE, Snyder-Heelan K, Livingstin, K. Adherence and attrition with intermittent and continuous exercise in overweight women. Int J Sports Med. 2003; 24: 459-64.

19. Slentz CA, Duscha BD, Johnson JL, Ketchum K, Aiken LB, Samsa GP, et al. Effects of the amount of exercise on body weight, body composition, and measures of central obesity: STRRIDE-a randomized controlled study. Arch Intern Med. 2004; 164: 31-9.

20. Rosenkilde M, Auerbach P, Reichkendler MH, Ploug T, Stallknecht BM, Sjodin A. Body fat loss and compensatory mechanisms in response to different doses of aerobic exercise- $-a$ randomized controlled trial in overweight sedentary males. Am J Physiol, Regul Integr Comp Physiol.2012; 303: 571-9.

21. Hansen $D$ et al. Continuous low- to moderate-intensity exercise training is as effective as moderate- to high-intensity exercise training at lowering blood $\mathrm{HbA}(1 \mathrm{c})$ in obese type 2 diabetes patients. Diabetologia. 2009; 52: 1789-97.

22. Shaw CS, Shepherd SO, Wagenmakers,AJ, ; Hansen D, Densale P, Van Loon LJ. Prolonged exercise training increases intramuscular lipid content and perilipin 2 expression in type I muscle fibers of patients with type 2 diabetes. Am J Physiol. 2012; 303: 1158-65.

23. Hansen D, Dendale P, Van Loon LJ, Meeusen R. The impact of training modalities on the clinical benefits of exercise intervention in patients with cardiovascular disease risk or type 2 diabetes mellitus. Sports Med. 2010; 40: 921-40.

24. Pavlou KN, Krey S, Stefee WP. Exercise as an adjunct to weight loss and maintenance in moderately obese subjects. Am J Clin Nutr. 1989; 49: 1115-23.

25. Keim NL, Barbieri TF, Van Loan MD, Anderson BL. Energy expenditure and physical performance in overweight women: response to training with and without caloric restriction. Metab. 1990; 39: 651-8.

26. Miller WC, Koceja DM, Hamilton EJ. A meta-analysis of the past 25 years of weight loss research using diet, exercise or diet plus exercise intervention. Int J Obes. Relat Metab Disord. 1997; 21: 941-7.

27. Volek JS et al. Effects of an 8-week weight-loss program on cardiovascular disease risk factors and regional body composition. Eur J Clin Nutr. 2002; 56: 585-92. 
28. Wallman K, Plant LA, Rakimov B, Maiorana AJ. The effects of two modes of exercise on aerobic fitness and fat mass in an overweight population. Res Sports Med. 2009; 17: 156-70.

29. Mediano MFF, Barbosa JSO, Moura AS, Willett WC, Sichieri R. A randomized clinical trial of home-based exercise combined with a slight caloric restriction on obesity prevention among women. Prev Med. 2010; 51: 247-52.

30. Wu T, Gao X, Chen M, Van Dam RM. Long-term effectiveness of diet-plus-exercise interventions vs. diet-only interventions for weight loss: a meta-analysis. Obes Rev. 2009; 10: 31323.

31. Muphy MH, Hardman AE. Training effects of short and long bouts of brisk walking in sedentary women. Med Sci Sports Exerc. 1998; 30: 152-7.

32. Schmidt WD, Biwer CJ, Kalscheuer LK. Effects of long versus short bout exercise on fitness and weight loss in overweight females. J Am College Nutr. 2001; 20: 494-501.

33. Jakicic JM, Wing RR, Butler BA, Robertson, RJ. Prescribing exercise in multiple short bouts versus one continuous bout: effects on adherence, cardiorespiratory fitness, and weight loss in overweight women. Int $\mathrm{J}$ Obes Relat Metab Disord.1995; 19: 893-901.

34. Thorton MK, Potteiger JA. Effects of resistance exercise bouts of different intensities but equal work on EPOC. Med Sci Sports Exerc. 2002; 34: 715-22.

35. Short KR, Sedlock DA. Excess postexercise oxygen consumption and recovery rate in trained and untrained subjects. $J$ Appl Physiol 1997; 83: 153-9.

36. Norman $A C$ et al. Influence of excess adiposity on exercise fitness and performance in overweight children and adolescents. Pediatrics. 2005; 115 : 690-1006.

37. Chaston TB, Dixon JB. Factors associated with percent change in visceral versus subcutaneous abdominal fat during weight loss: findings from a systematic review. Int J Obes. 2008; 32: 619-28.

38. Goodrick GK, Poston WS, Foreyt JP. Methods for voluntary weight loss and control: update 1996. Nutrition. 1996; 12: 672-6.
39. Hill JO, Drougas H, Peters JC. Obesity treatment: can diet composition play a role? Ann Intern Med.1993; 119: 694-7.

40. Perri MG, Nezu AM, Patti ET, Mccann KL. Effect of length of treatment on weight loss. J Consult Clin Psychol. 1989; 57: 450-2.

41. Foster GD, Makris AP, Bailer BA. Behavioral treatment of obesity. Am J Clin Nutr. 2005; 82: 230-5.

42. Lowe MR, Miller-kovach K, Phelan S. Weight-loss maintenance in overweight individuals one to five year following successful completion of a commercial weight loss program. Int J Obes. Relat Metab Disord. 2001; 25: 325-31.

43. Powell L, Calvin JEIII, Junior Calvin JE. Effective obesity treatments. Am Psychol. 2007; 62: 234-46.

44. Wadden TA, Butryn ML. Behavioral treatment of obesity. Med Clin North Am 2000; 32: 981-1003.

45. Borsheim E, Bahr R. Effect of exercise intensity, duration and mode on post-exercise oxygen consumption. Sports Med. 2003; 33: 1037-60.

46. Kaminsky LA, Padjen S, Laham-Saeger J. Effect of split exercise sessions on excess post-exercise oxygen consumption. Br J Sports Med. 1990;24: 95-8.

47. Gore CJ, Withers RT. The effect of exercise intensity and duration on the oxygen deficit and excess post-exercise oxygen consumption. Eur J Appl Physiol Occup Physiol. 1990; 60: 169-74.

48. Haskell WL et al. Physical Activity and Public Health: Updated Recommendation for Adults From the American College of Sports Medicine and the American Heart Association. Med Sci Sports Exerc. 2007; 39: 1423-34.

49. Andersen RE. Exercise, an Active Lifestyle, and Obesity: Making the Exercise Prescription Work. Phys Sportsmed 1999; 27: 41-8.

50. Brownell, KD. Diet, exercise and behavioural intervention: the nonpharmacological approach. Eur J Clin Invest 1998; 28 : 19-21. 